\title{
Drawing the line between commensal and pathogenic Gardnerella vaginalis through genome analysis and virulence studies
}

\author{
Michael D Harwich Jr'1, Joao M Alves ${ }^{1}$, Gregory A Buck1', Jerome F Strauss III2, Jennifer L Patterson2, Aminat T Oki1, \\ Philippe $\mathrm{H}$ Girerd ${ }^{2}$ and Kimberly K Jefferson*1
}

\begin{abstract}
Background: Worldwide, bacterial vaginosis (BV) is the most common vaginal disorder. It is associated with risk for preterm birth and HIV infection. The etiology of the condition has been debated for nearly half a century and the lack of knowledge about its cause and progression has stymied efforts to improve therapy and prevention. Gardnerella vaginalis was originally identified as the causative agent, but subsequent findings that it is commonly isolated from seemingly healthy women cast doubt on this claim. Recent studies shedding light on the virulence properties of $G$. vaginalis, however, have drawn the species back into the spotlight.

Results: In this study, we sequenced the genomes of a strain of G. vaginalis from a healthy woman, and one from a woman with bacterial vaginosis. Comparative analysis of the genomes revealed significant divergence and in vitro studies indicated disparities in the virulence potential of the two strains. The commensal isolate exhibited reduced cytotoxicity and yet the cytolysin proteins encoded by the two strains were nearly identical, differing at a single amino acid, and were transcribed at similar levels. The BV-associated strain encoded a different variant of a biofilm associated protein gene and demonstrated greater adherence, aggregation, and biofilm formation. Using filters with different pore sizes, we found that direct contact between the bacteria and epithelial cells is required for cytotoxicity.

Conclusions: The results indicated that contact is required for cytotoxicity and suggested that reduced cytotoxicity in the commensal isolate could be due to impaired adherence. This study outlines two distinct genotypic variants of $G$. vaginalis, one apparently commensal and one pathogenic, and presents evidence for disparate virulence potentials.
\end{abstract}

\section{Background}

G. vaginalis has had a checkered taxonomic beginning. It was originally isolated by Leopold [1] and later associated with the vaginal disorder now referred to as bacterial vaginosis and named Haemophilus vaginalis by Gardner and Dukes [2,3]. Subsequently, metabolic requirements and gram staining led to its reclassification within the genus Corynebacterium. Greenwood and Pickett suggested that the organism did not belong in this genus either and that it be placed in its own genus, named after its discoverer [4]; a contention later supported by DNA-DNA hybridization [5]. Gardnerella is in the Family Bifidobacteri-

\footnotetext{
* Correspondence: kkjefferson@vcu.edu

1 Department of Microbiology and Immunology, Medical College of Virginia Campus of Virginia Commonwealth University, 1101 E. Marshall Street - PO Box 980678, Richmond, VA 23298 USA

Full list of author information is available at the end of the article
}

aceae and is most closely related to species in the Genus Bifidobacterium. The cells are small, nonmotile pleomorphic rods, which may be piliated. The length of the rods and gram staining vary depending on the growth medium [6]. Electron microscopy and the lack of lipopolysaccharide production demonstrates that the cell wall is grampositive, although the peptidoglycan layer can be thinner than many gram-positive organisms, resulting in negative gram staining [7]. G. vaginalis is a fastidious organism and requires complex medium for growth. Studies using metabolic methods of identification indicate that it is catalase-negative, exhibits $\alpha$-glucosidase activity, starch hydrolysis, hippurate hydrolysis, acid phosphatase activity, but lacks gelatin and esculin hydrolysis, and salt tolerance [6]. G. vaginalis is anaerobic and can utilize the carbohydrates dextrin, fructose, glucose, maltose, ribose, 
starch, and sucrose through fermentation. Some strains ferment mannose, galactose, and sucrose, and a few strains ferment xylose and trehalose but it does not ferment mannitol, raffinose, rhamnose, or sorbitol [6].

Despite the fact that bacterial vaginosis (BV) is the leading vaginal disorder globally, very little is known about its etiology or pathogenesis. It fails to conform to any of Koch's postulates; it is not associated with a single bacterial species, no single species has ever been found to reliably elicit the disorder in healthy women, and the adaptation of the bacterial species involved to life in the human host has precluded the development of a useful animal model. Gardner and Dukes identified G. vaginalis as the etiologic agent but findings that pure cultures did not always cause BV drew this allegation into question $[3,8]$. Subsequent studies analyzed the role of additional species such as Atopobium vaginae and Mycoplasma hominis, but efforts have yet to unequivocally establish the role of a single species $[9,10]$. Recent studies of the virulence properties of $G$. vaginalis have revealed its ability to adhere avidly to and establish a tenacious biofilm on the vaginal epithelium in women with BV and have characterized the molecular basis for its cytotoxicity $[11,12]$. These virulence studies along with findings that G. vaginalis is the only species that can be detected in the vast majority of cases of BV and increasing reports of extravaginal infections in which it is the only species isolated, such as bacteremia and osteomyelitis, have drawn this species back into the spotlight and resurrected the notion of its pathogenic potential [13-16].

The role of $G$. vaginalis in BV has also been disputed because the organism can inhabit the genital tract of healthy women $[17,18]$. However, the numbers of G. vaginalis on the vaginal epithelium of healthy women are several logs lower than the numbers found in women with BV $[11,18,19]$. In addition, it has been reported recently that the biotypes of $G$. vaginalis isolated from healthy women differ from those isolated from women with BV [20]. The lack of genetic characterization of this organism leaves open the possibility that distinct pathogenic and non-pathogenic strains or even subspecies exist. The Human Microbiome Project, an effort supported by the $\mathrm{NIH}$, will soon produce a tremendous amount of genetic information about the bacteria associated with BV and efforts are underway to sequence strains of G. vaginalis, A. vaginae, and Mobiluncus. In an effort to better understand the physiology and pathogenic potential of G. vaginalis, we performed whole genome sequence analysis of an isolate from a case of BV (G. vaginalis strain AMD), and to determine whether isolates may vary in their pathogenic potential, we also performed whole genome sequence analysis of an isolate from a healthy woman ( $G$. vaginalis strain $5-1$ ). We analyzed the virulence potential of these two strains using a series of in vitro assays to quantify their cytotoxic activities, their ability to adhere to cervical epithelial cells, and their capacity for biofilm formation.

\section{Results}

\section{Whole genome sequence analysis}

Pyrosequencing yielded high coverages of the BV-associated G. vaginalis strain, 5-1 (accession number: ADAM00000000) and the healthy subject isolate, AMD (accession number: ADAN00000000) genomes $(\sim 175 \mathrm{X}$ and $\sim 130 \mathrm{X}$, respectively). The data for both genomes assembled into approximately 20 contigs greater than 500 bp. Both genomes were estimated at approximately 1.65 Mb. In a MUMmer alignment of the genomic sequences, the minimum percent identity between the two strains was $76.66 \%$ and the maximum was $99.07 \%$, with an overall average of $93.62 \%$. GBrowse sites were designed for strains 5-1 and AMD http://www.gardnerella.mic.vcu.edu. Overall GC content figures were $41.95 \%$ (5-1) and $42.08 \%$ (AMD), and the numbers of protein coding genes predicted were 1,340 (5-1) and 1,318 (AMD). There was only one complete assembled copy of the ribosomal RNA gene cluster, suggesting that all copies are identical or close to it. Examination of contig boundaries suggests that there are at least two and probably three or four copies of the rRNA gene cluster. The number of predicted tRNAs was identical in the two strains: 45 .

\section{Metabolic pathways in the two strains}

Analysis of the metabolic potential yielded interesting insights into the basis for the complex growth requirements of this fastidious organism [21]. Metabolic pathways for amino acid synthesis are absent, apart from a few possible short conversions including fumarate to Laspartate. Pathways for O-glycan, N-glycan, peptidoglycan, and lipopolysaccharide biosynthesis seemed to be well populated with the required enzymes. In addition, many of the required enzymes that convert L-amino acids to D-amino acids were identified. The portions of the pentose phosphate pathway allowing synthesis of the required precursors of nucleotide biosynthesis were also present. Purine and pyrimidine biosynthesis pathways were represented by a number of enzymes, permitting synthesis of several (but not all) bases and nucleotides. Interestingly, both strains appear to lack two enzymes that are essential for glycolysis; phosphofructokinase, and fructose-bisphosphate aldolase. If the enzymes are truly absent, this deficiency could be partially compensated by the pentose phosphate pathway. Another possibility is that $G$. vaginalis performs these functions using novel enzymes that were not identified in our similarity searches. 


\section{Putative virulence factors in both strains}

The only characterized virulence factor in G. vaginalis is vaginolysin [12]. Vaginolysin is a cholesterol-dependent cytolysin that may contribute to virulence by making cellular contents more available as a substrate for bacterial growth. This cytolytic action may also have the coincidental effect of making the vaginal epithelium more permeable to HIV virions. The gene for vaginolysin, $v l y$, in strain 5-1 exhibited $89 \%$ identity at the nucleotide level and $94 \%$ identity at the amino acid level to vly from $G$. vaginalis strains 14019, 14018, and 49145 [12]. Vly from strains 5-1 and AMD were 99\% identical at the amino acid level, differing at a single amino acid (T35A).

Analysis of the $G$. vaginalis genome revealed a number of additional putative virulence factors, including a gene with similarity to the P30/P32 adhesins (GV51_0007, GVAMD_0260). These adhesins are produced by species of Mycoplasma and are involved in adherence to human tissues [22]. Previous reports have demonstrated that fresh clinical isolates of G. vaginalis are piliated, but suggest that pilus production may be lost upon in vitro passage of the bacteria [23,24]. However, even though loci likely to be involved in pilus assembly were present (GV51_0383 - 0387, GVAMD_1118 - 1122), we were unable to identify pili by transmission electron microscopy of strains 5-1 and AMD, even when the bacteria when in vitro culture was restricted (Fig. 1).

\section{Proteins encoded in only one strain}

OrthoMCL analysis was performed to identify proteins putatively present in only one strain (Table 1). A number of these were small, hypothetical proteins that could represent pseudogenes and several did not exhibit significant similarity to any proteins of known function. Strain 5-1 contained a number of putative phage proteins that were absent in AMD. Strain AMD contained a number of putative serine-threonine kinases not present in 5-1, however the role of these kinases was unclear from similarity searches. AMD also contained an apparent toxin-

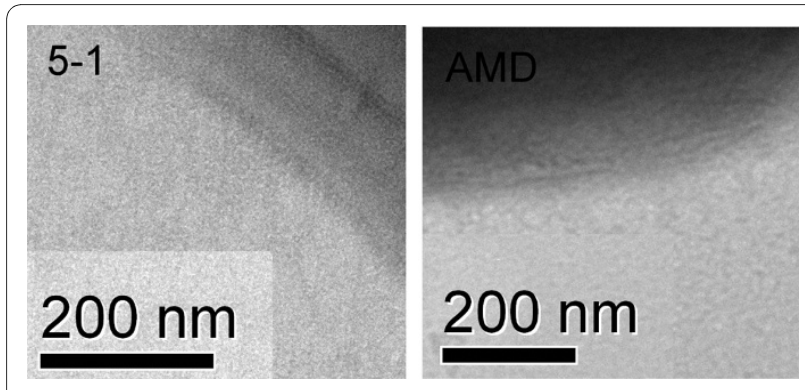

Figure 1 Pili were not detectable on strains AMD and 5-1. Tandem Electron Microsopy was used to investigate pilus expression by the two strains. The cell surfaces are shown at high resolution and suggest the absence of pilus production under the conditions used in this study. antitoxin gene cassette. Interestingly, the antitoxin protein exhibited $100 \%$ identity to a protein from $A$. vaginae and $65 \%$ identity to a protein from $M$. mulieris suggesting that horizontal gene transfer occurs between BV-associated bacterial species.

\section{Putative antibiotic resistance determinants}

BV can be difficult to treat and the rate of relapse or recurrence is $50 \%$ within a year [25]. Resistance to metronidazole, the drug of choice for BV therapy, has been seen in some strains of $G$. vaginalis, as has resistance to tetracycline [26]. We tested strains 5-1 and AMD for in vitro susceptibilities to 13 different antibiotics (Table 2). Both strains were sensitive to tetracycline $(\mathrm{MIC}=0.5 \mu \mathrm{g}$ / $\mathrm{mL}$ ) and appeared to lack the tet $M$ gene, found in tetracycline-resistant strains of G. vaginalis [27].

Therapeutically achievable levels of metronidazole are generally effective against bacteria with an $\mathrm{MIC}<=8 \mu \mathrm{g} /$ $\mathrm{mL}$, whereas those with an MIC $=16 \mu \mathrm{g} / \mathrm{mL}$ are considered intermediate resistant and bacteria with an $\mathrm{MIC}>=$ $32 \mu \mathrm{g} / \mathrm{mL}$ are considered resistant [28]. Clinical cure rates of $75 \%$ have been reported for oral metronidazole therapy [29]. Both strains exhibited intermediate resistance with MICs of $19.5 \mu \mathrm{g}$ metronidazole/ml. The most well-characterized mechanism of resistance to metronidazole is inactivation or deletion of genes with nitroreductase activity [30]. Both strains had nitroreductase genes (GV51_0575, GV51_0595, GVAMD_0064, and GVAMD_0125) but the expression of the genes and functionality of the proteins was not determined in this study.

Nalidixic acid resistance in G. vaginalis has been well established and strains 5-1 and AMD both exhibited resistance with an MIC above $300 \mu \mathrm{g} / \mathrm{ml}$. The ORFs GV51_0431 and GVAMD_1169, which are 98\% identical, are predicted to encode a protein with similarity to multidrug efflux pumps in the MATE (multidrug and toxin exclusion) family. Members of this family include Staphylococcus aureus MepA, which has been shown to confer fluoroquinolone resistance so this may be involved in resistance to nalidixic acid (18).

The strains also exhibited intermediate resistance to kanamycin. Both genomes contained a gene for a phosphotransferase enzyme family (GV51_0090, GVAMD_0184; 95\% identical). This family includes proteins that inactivate certain aminoglycosides such as kanamycin. Therefore it is possible that the product of this gene plays a role in permitting kanamycin resistance in $G$. vaginalis.

The genomes also encoded an EmrB/QacA family protein (GV51_1060, GVAMD_0885; 99\% identical), which could confer resistance to certain streptogramins, however, the strains were both susceptible to clindamycin and erythromycin, although strain 5-1 was more sensitive to erythromycin than was strain AMD. 
Table 1: ORFs unique to one or the other G. vaginalis strains.

\begin{tabular}{|c|c|c|c|c|c|}
\hline $\begin{array}{l}\text { Unique to } \\
\text { strain }\end{array}$ & Gene Identifier & Predicted function/homologues & $\begin{array}{l}\text { Unique to } \\
\text { strain }\end{array}$ & Gene Identifier & $\begin{array}{l}\text { Predicted function/ } \\
\text { homologues }\end{array}$ \\
\hline \multirow[t]{17}{*}{ AMD } & Gv1_0004 GVAMD_0044 & No significant similarity & $5-1$ & Gv1_0006 GV51_0275 & Hypothetical protein \\
\hline & Gv1_0004 GVAMD_0852 & No significant similarity & & Gv1_0006 GV51_0972 & Hypothetical protein \\
\hline & Gv1_0004 GVAMD_1243 & Hypothetical protein & & Gv1_0006 GV51_1019 & Hypothetical protein \\
\hline & Gv1_0004 GVAMD_0114 & No significant similarity & & Gv1_0008 GV51_0361 & Site specific recombinase \\
\hline & Gv1_0005 GVAMD_0177 & Serine/threonine protein kinase & & Gv1_0008 GV51_1208 & Phage-like integrase \\
\hline & Gv1_0005 GVAMD_1234 & Serine/threonine protein kinase & & Gv1_0009 GV51_0427 & $\begin{array}{l}\text { Conserved hypothetical } \\
\text { protein }\end{array}$ \\
\hline & Gv1_0005 GVAMD_1232 & $\begin{array}{l}\text { Serine/threonince protein } \\
\text { kinase }\end{array}$ & & Gv1_0009GV51_1210 & $\begin{array}{l}\text { Conserved hypothetical } \\
\text { protein }\end{array}$ \\
\hline & Gv1_0005 GVAMD_0389 & $\begin{array}{l}\text { Serine/threonince protein } \\
\text { kinase }\end{array}$ & & Gv1_0010GV51_0968 & Phage associated protein \\
\hline & Gv1_0011 GVAMD_0742 & $A B C$ transporter & & Gv1_0010 GV51_0974 & $\begin{array}{l}\text { Conserved hypothetical } \\
\text { protein }\end{array}$ \\
\hline & Gv1_0011 GVAMD_0743 & $A B C$ transporter & & Gv1_0012 GV51_0058 & $\begin{array}{l}\text { Type } 1 \text { restriction } \\
\text { modification system }\end{array}$ \\
\hline & Gv1_0016 GVAMD_0086 & No significant similarity & & Gv1_0012 GV51_0345 & $\begin{array}{l}\text { Type } 1 \text { restriction } \\
\text { modification system }\end{array}$ \\
\hline & Gv1_0016 GVAMD_0521 & No significant similarity & & Gv1_0013 GV51_0141 & $\begin{array}{l}\text { Conserved hypothetical } \\
\text { protein }\end{array}$ \\
\hline & Gv1_0017 GVAMD_0113 & Hypothetical protein & & Gv1_0013 GV51_0412 & Cell wall hydrolase \\
\hline & Gv1_0017 GVAMD_0456 & Hypothetical protein & & Gv1_0014 GV51_0363 & Cell wall protein \\
\hline & Gv1_0018 GVAMD_0143 & $\begin{array}{l}\text { Conserved hypothetical, } \\
\text { putative anti-toxin cassette }\end{array}$ & & Gv1_0014 GV51_1225 & Hypothetical protein \\
\hline & Gv1_0018 GVAMD_0681 & Conserved hypothetical & & Gv1_0015 GV51_1339 & $\begin{array}{l}\text { Conserved hypothetical } \\
\text { protein }\end{array}$ \\
\hline & Gv1_0019 GVAMD_0390 & 2-component regulator & & Gv1_0015 GV51_1340 & $\begin{array}{l}\text { Conserved hypothetical } \\
\text { protein }\end{array}$ \\
\hline
\end{tabular}

Gv1_0019 GVAMD_0535 Hypothetical, beta-lactamase class A 
Table 1: ORFs unique to one or the other G. vaginalis strains. (Continued)

\begin{tabular}{cl}
\hline Gv1_0020 GVAMD_0448 & Hypothetical protein \\
\hline Gv1_0020 GVAMD_0928 & Conserved hypothetical protein \\
\hline Gv1_0021 GVAMD_0931 & Hypothetical protein \\
\hline Gv1_0021 GVAMD_1004 & Hypothetical protein \\
\hline
\end{tabular}

\section{Analysis of surface structures}

Because we had noted a difference in the level of aggregation and biofilm formation of the two strains when they were cultured in $10 \%$ human serum (sBHIs), we analyzed the surface of the bacteria by transmission electron microscopy. We observed a layer that resembled a polysaccharide capsule on the surface of strain 5-1 that was absent on AMD (Fig. 2).

\section{Cytotoxicity}

Despite the finding that the $v l y$ genes of AMD and 5-1 encode nearly identical proteins, with only a single amino acid mismatch, cytotoxicity assays indicated that after 1 hour AMD, the BV-isolate, induced rounding and other cytopathologic changes in a monolayer of ME-180 cervical epithelial cells (data not shown), and complete lysis of the monolayer by 3 hours (Fig. 3B). In contrast, ME-180 cells exposed to the non-BV isolate, 5-1, were still attached and exhibited normal spreading epithelial morphology after 1 hour (data not shown) and had only begun to exhibit rounding and other cytopathogenic changes by 3 hours (Fig. 3C). Strain 5 -1 led to complete disruption of the ME-180 monolayer after 12 hours (data

Table 2: In vitro susceptibilities of G. vaginalis strains to 12 antimicobial agents.

\begin{tabular}{|c|c|c|}
\hline \multirow[t]{2}{*}{ Antibiotic } & \multicolumn{2}{|c|}{ MIC ( $\mu \mathrm{g} / \mathrm{mL})$} \\
\hline & Strain 5-1 & Strain AMD \\
\hline Ampicillin & 0.125 & 0.125 \\
\hline Nafcillin & 0.12 & 0.12 \\
\hline Ciprofloxacin & 1 & 2 \\
\hline Nalidixic acid & 390 & 390 \\
\hline Chloramphenicol & 0.5 & 1 \\
\hline Minocycline & 0.156 & 0.078 \\
\hline Tetracycline & 0.5 & 0.5 \\
\hline Erythromycin & 0.032 & 0.128 \\
\hline Clindamycin & 0.625 & 0.625 \\
\hline Kanamycin & 32 & 32 \\
\hline Rifampin & 0.063 & 0.063 \\
\hline Metronidazole & 19.5 & 19.5 \\
\hline
\end{tabular}




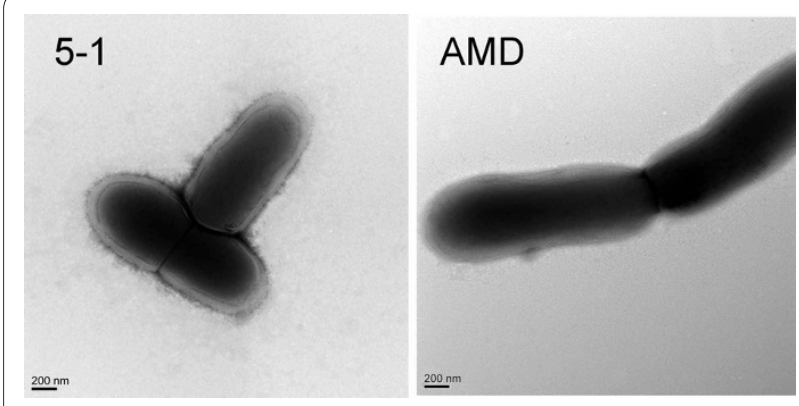

Figure 2 Transmission Electron Microscopy of G. vaginalis strains. The left panel is 5-1 while the panel on the right is AMD. When the strains were grown in the presence of $10 \%$ human serum, a capsulelike material was present on 5-1, but was undetectable on AMD.

not shown) suggesting that Vly from this strain was functional. To determine if differences in expression of $v l y$ could account for the reduced cytotoxicity associated with the commensal strain, we compared the vly promoter regions of the two strains and found that 5-1 was lacking $2 \mathrm{bp}$ that were present in the AMD vly promoter. However, quantitative realtime RT-PCR indicated that transcriptional expression of the $v l y$ gene was equivalent in the two strains (data not shown).

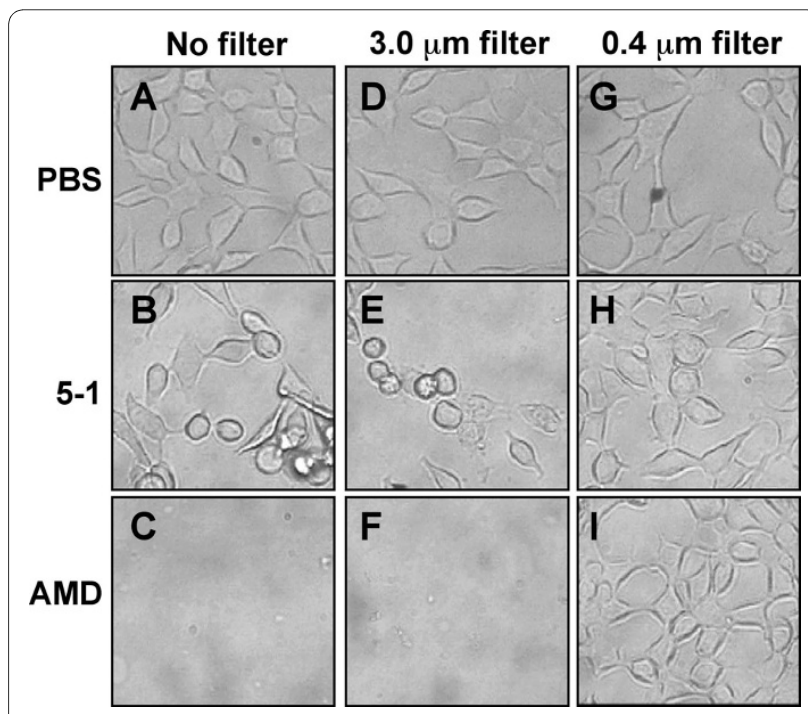

Figure $\mathbf{3}$ Cytotoxicity caused by $\mathbf{G}$. vaginalis strains. Equal numbers of either AMD, 5-1, or PBS control were either added to a ME-180 monolayer directly (A-C), or were loaded into transwell filters with either $3 \mu \mathrm{m}(\mathrm{D}-\mathrm{F})$ or $0.4 \mu \mathrm{m}(\mathrm{G}-\mathrm{I})$ pore-size. The cells were monitored microscopically during the incubation for phenotypic alterations associated with toxic effects. After incubating the ME-180 cells with the bacteria for 3 hours, strain AMD had caused complete lysis of the monolayer whereas the cells incubated with strain 5-1 had just begun to exhibit rounding. The cytotoxic effects were completely blocked by the transwell with $0.4 \mu \mathrm{m}$ pore size, which did not allow contact between the ME-180 s and the bacteria while the $3 \mu \mathrm{m}$ pores, which did allow contact, permitted the cytotoxic effects.

\section{Adherence to epithelial cells}

We analyzed the ability of the two G. vaginalis strains to adhere to cultured ME-180 cervical epithelial cells was assayed by confocal microscopy. While equal amounts of the two strains were added to ME-180 monolayers, adherence of strain AMD was much more pronounced relative to that of the non-BV isolated strain 5-1 (Fig. 4). It was also observed that strain AMD was more aggregative than was strain 5-1. This suggests that the capability of $\mathrm{BV}$ isolates to bind to and adhere to vaginal epithelium may be higher than non-BV isolates. As pilus expression could not be demonstrated in either strain, we searched for other putative adhesins. Both strains harbored a biofilm associated protein (BAP) family gene (Fig. 5A). BAP homologues in other species are involved not only in biofilm formation, but in adherence to epithelial cells as well, so we compared the sequences of the BAP genes encoded by 5-1 and AMD. ClustalW alignment of the amino acid sequences of the BAP family protein encoded by AMD and 5-1 showed considerable sequence differences between the two proteins (Fig. 5B). The BAP homologues had a highly repetitive structure as is characteristic of the other BAP family proteins (Fig. 5A) and a majority of the sequence differences between the two strains were located within the region containing the $\mathrm{B}, \mathrm{C}$, and $\mathrm{D}$ repeats. BAP is also predicted to contain several Rib domains. Rib domains were named after the Rib protein of Group B Streptococcus, an immunogenic surface proteins with a highly repetitive structure [31]. The number and distribution of Rib domains in the BAP homologues differed between AMD and 5-1.

\section{Role of adherence in cytotoxicity}

Other cholesterol-dependent cytolysins depend upon adherence to exert their activity [32]. Because we observed that AMD exhibited increased adherence, we investigated the possibility that this was related to the greater cytotoxic activity associated with AMD. G. vagi-
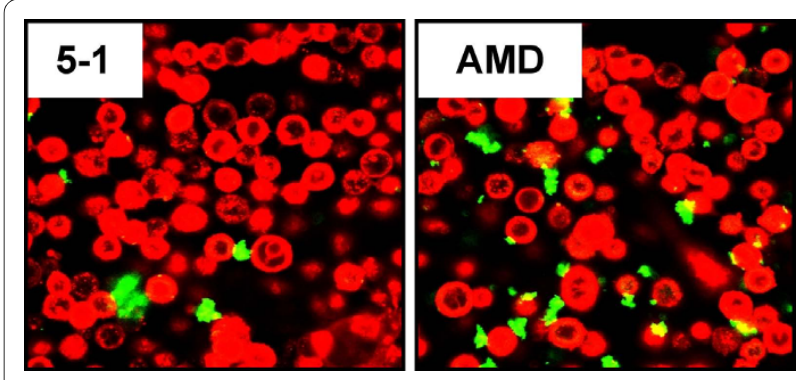

Figure 4 Adherence of G. vaginalis to cultured vaginal epithelium. Equal amounts of the indicated strains of $G$. vaginalis (green) were added to ME-180 cells (red). The cells were stained with BacLight green and Vybrant Red stains respectively. Adherence was analyzed by confocal microscopy following incubation and extensive washing with $1 \mathrm{X}$ PBS. 


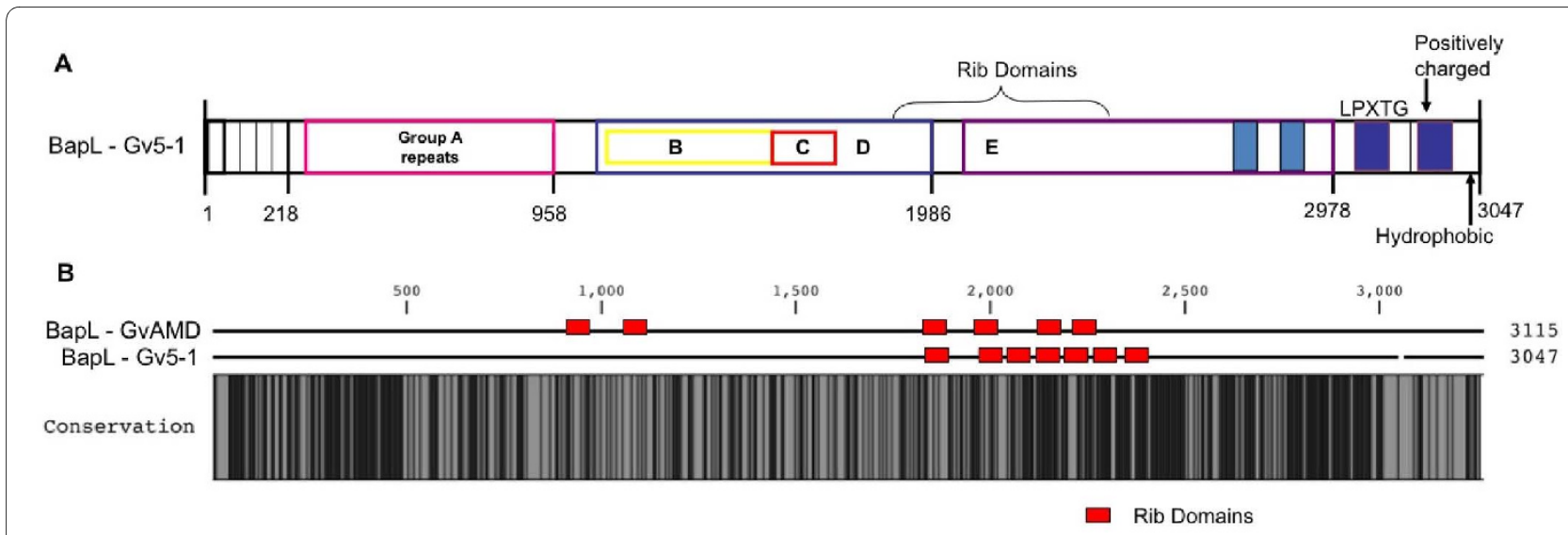

Figure 5 BAP domain structure and inter-strain conservation. A) Diagram representing the repeat structures found within the BAP encoded by G. vaginalis strain 5-1. Repeats units are diagramed above and named based upon the similarity to Bap proteins from other species. B) A ClustalW alignment of BAPs from strains AMD and 5-1 is shown, black represents 100\% conservation. Rib domains are indicated by red boxes.

nalis was added to transwell filters with a pore-size equal to either $3 \mu \mathrm{m}$, to allow the bacteria to penetrate the filter and make contact with the monolayer, or $0.4 \mu \mathrm{m}$, to prevent direct contact of the bacteria and monolayer but to allow proteins to flow through to the ME-180 s. We found that direct contact with the monolayer was required for cytotoxic activity (Fig. 3D-I), suggesting that the disparity in the adherence of the two strains could account for differences in cytotoxic activity.

\section{Capacities for biofilm-formation}

The ability to form a biofilm is a marker of potential virulence and a cause of recurrent infections. Since BAP proteins are known to play a role not only in adherence but in biofilm formation in other species [33], we chose to investigate whether the disparity in the sequences and expression of the BAP homologues could translate into differences in biofilm forming activity. Safranin staining of biofilms (Fig. 6A) produced by G. vaginalis strains AMD and 5-1 after 24 hours of growth showed that the BV-isolate AMD produced a thicker biofilm. Quantitative measurement of biofilm growth as percentage of total growth indicated that AMD had a significantly greater biofilm-forming capacity than the non-BV isolate 5-1 (Fig. 6B). The relationship between biofilm capacity and association with BV was further investigated using additional strains. We found that two other G. vaginalis BVisolates, strains 101 and 551 produced similar levels of biofilm growth as did strain AMD and significantly more than strain 5-1. A second healthy isolate, strain 465, showed significantly less biofilm growth than the BV-isolates (Fig. 6B). These data suggest that BV-isolates tend to produce more biofilm growth than do non-BV associated isolates of G. vaginalis, which would reasonably be consistent with greater virulence.

\section{Discussion}

G. vaginalis can be isolated from the majority of BV cases but it can also be isolated, albeit at much lower numbers, from healthy women. Very little is currently known about the genetic composition of G. vaginalis, the diversity of strains or about its physiology. It has been reported that

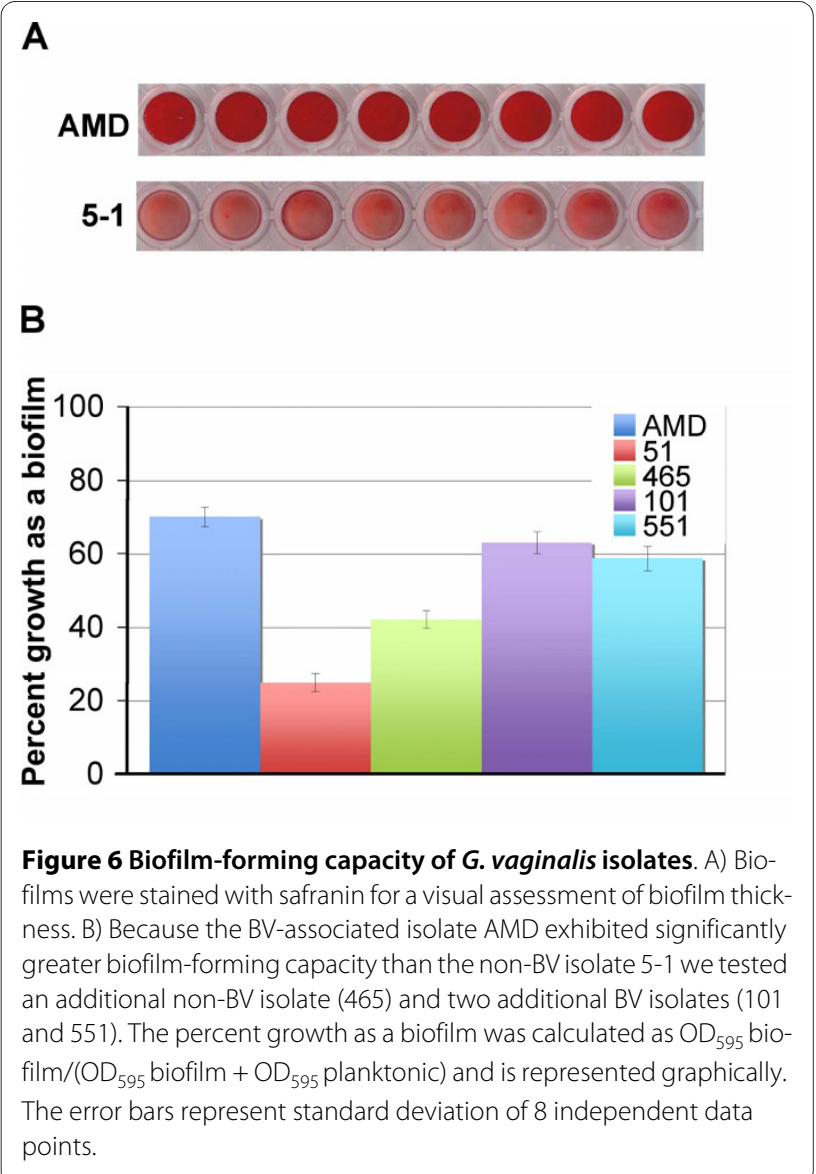


certain biotypes of G. vaginalis are more frequently associated with BV [20]. In order to investigate the possibility that certain strains are more likely to be associated with $\mathrm{BV}$, we sequenced a BV-associated strain and a healthy isolate and characterized their virulence potentials with a series of in vitro assays for cytotoxicity, adherence, and biofilm formation. We found that the BV-associated strain was significantly more cytotoxic than the non-BV isolate suggesting that differing levels of cytotoxicity may be related to the propensity of a strain to cause disease. Proteins encoded by the $v l y$ genes differed by only a single amino acid and the promoters differed by only $2 \mathrm{bp}$. The disparity in the level of cytotoxicity of the two strains could be related to the amino acid substitution, but the residue is not near the active site or other amino acids known to play a key role in function [12]. The differences in cytotoxicity are likely a function of adherence. We found that strain AMD adheres more avidly to cultured vaginal epithelial cells than does the non-BV isolate, 5-1. Therefore it is possible that the increase in cytotoxicity seen with strain AMD is simply due to its ability to adhere and thereby deliver vaginolysin more directly to the eukaryotic monolayer. Consistent with this hypothesis was the finding that a $0.4 \mu \mathrm{M}$ filter effectively blocked G. vaginalis-mediated cytotoxicity. If the toxic effector molecule produced by the bacteria was freely secreted in sufficient concentrations and the secreted form was cytotoxic, then phenotypic changes of the ME- 180 monlayer would have been seen even under conditions that prevented direct cell to cell contact, i.e. the $0.4 \mu \mathrm{M}$ filter. However the filter ablated cytotoxicity in ME-180 s suggesting that direct contact between G. vaginalis and the vaginal epithelium is required for effective delivery of the $v l y$ gene product, as is the case with other cholesteroldependent cytolysins [32].

These results have also demonstrated a clear difference in the ability of various strains to adhere to cultured cervical epithelial cells. While both AMD and 5-1 were able to adhere to the ME- 180 monolayer, AMD exhibited heightened adherence. Adherence is a prerequisite for infection, and these results suggest that BV isolates are able to adhere, and thus establish an infection much more readily than non-BV isolates. Factors influencing the ability of a given strain to adhere to eukaryotic cells would be governed by the proteins and structures present upon the bacterial cell surface and the interaction between those factors and the ME-180 surface proteins and structures. We found a gene encoding a biofilm associated protein (BAP) family protein. BAP proteins are large, cell wallanchored adhesins that can mediate both adherence to host cells and intercellular adherence, which contributes to biofilm formation [33,34]. Interestingly, the gene sequences for AMD and 5-1 BAP were quite disparate.
This was particularly noticeable in the repeat regions, the region of BAP proteins that generally mediates adherence. We also noted that the healthy isolate appeared to be coated in a capsular structure, whereas the BV-associated isolated did not appear to express this structure. This may negatively impact adherence to vaginal epithelial cells, or biofilm formation. Pneumococci produce a capsule that has been linked to a decrease in biofilm formation supporting this hypothesis [35]. Both genomes contained multiple potential operons for capsular polysaccharide biosynthesis although there was significant divergence in the proteins encoded by the two strains.

We also found a significant difference in the propensity of the two strains to form a biofilm in vitro. Biofilm formation by $G$. vaginalis has recently been implicated in BV [11]. The ability for a strain to grow as a biofilm would likely confer resistance to mucosal immune defenses and antibiotic resistance, which could contribute to initial and recurrent colonization. Furthermore, lactobacilli normally associated with the healthy vagina produce byproducts such as lactic acid and hydrogen peroxide that normally suppress the growth of anaerobes such as $G$. vaginalis, but biofilm formation leads to increased resistance to these byproducts [36]. Therefore, biofilm formation may enable proliferation of G. vaginalis even in the presence of lactobacilli. Finally, biofilm formation is associated with increased antibiotic resistance and appears to play a role in treatment failure and recurrence in cases of BV [37]. The difference in biofilm forming capacity between these two strains could be related to the differences between the respectively encoded BAPs. The differences in domain distribution within the central repeat region and, potentially more importantly, the number and distribution of the Rib domains and repeats in strain AMD could better promote adherence and/or bacterial aggregation. Furthermore, our results were suggestive of a correlation between greater biofilm-forming capacity and association with BV.

This work clearly demonstrates strain differences between G. vaginalis isolates that could impact the ability of this organism to cause disease. The precise role for $G$. vaginalis in BV pathogenesis is still unclear, but this study suggests an explanation for the presence of this organism in the absence of BV. A limitation of this study is the restricted number of strains studied. Advances in sequencing technology and the Human Vaginal Microbiome Projects, taking place at Virginia Commonwealth University and the University of Maryland, will lead to the analysis of additional strains, which will reveal further insight into the role of the genetic background of G. vaginalis strains in pathogenesis and to clearly demarcate the differences between strains that are directly related to the propensity to cause disease. 


\section{Conclusions}

This study provides evidence to support the hypothesis that certain biotypes of G. vaginalis are unable or unlikely to cause disease while other strains are better suited to elicit disease. The results suggest that the strains that fail to elicit BV may not be able to adhere to, or form a biofilm on the vaginal epithelium as avidly as strains that cause BV. Results from genomic sequence analysis identified significant differences between a strain associated with BV and a strain from a healthy subject. The sequencing data reveals genes that may be involved in virulence and opens up avenues for further study of the pathogenesis of BV.

\section{Methods}

\section{Gardnerella strains and growth conditions}

Gardnerella vaginalis strains 5-1 and 465 were originally isolated from two healthy women without BV as diagnosed by the Nugent gram stain scoring system and strains 101 and 551 were isolated from women diagnosed with BV at Brigham and Women's Hospital, Boston, MA [38]. Strain AMD was isolated from a woman diagnosed with BV based on Amsel criteria at VCU Women's Health Clinic [39]. The strains were grown in Brain Heart Infusion (BHI) broth (EMD, Gibbstown, $\mathrm{NJ}$ ) at $37^{\circ} \mathrm{C}$ using the AnaeroPack system (Mitsubishi Gas Chemical Co, Tokyo, Japan).

\section{DNA isolation}

G. vaginalis strains were grown in $200 \mathrm{~mL}$ BHI overnight. The cells were collected by centrifugation, resuspended in $7 \mathrm{~mL}$ TNE buffer $(10 \mathrm{mM}$ Tris $\mathrm{HCl} \mathrm{pH} 8.0,10 \mathrm{mM}$ $\mathrm{NaCl}, 10 \mathrm{mM}$ EDTA) containing $1 \%$ Triton X-100, and 10 $\mathrm{mg}$ lysozyme $/ \mathrm{mL}$, and incubated at $37^{\circ} \mathrm{C}$ for 30 minutes. Proteinase $\mathrm{K}(0.3 \mathrm{mg} / \mathrm{mL})$ was added and the suspension was incubated for 1 hour at $65^{\circ} \mathrm{C}$. DNA was extracted with $7 \mathrm{~mL}$ 1:1 phenol/chloroform, followed by $7 \mathrm{~mL}$ chloroform, and precipitated with $1 / 10$ volume $3 \mathrm{M}$ sodium acetate and 2 volumes ethanol.

\section{Sequencing}

Sequencing was performed using Roche 454 technology. One full run of GS FLX and one half run GS FLX XLR were done for strain 5-1 (coverage of $\sim 175 \mathrm{X}$ ), while one half run of GS FLX XLR was done for strain AMD ( 130 $x$ coverage). Five micrograms of each strain's DNA was sequenced as per the standard Roche sequencing protocol.

\section{Sequence analysis}

Genomes were assembled by Roche's software Newbler 2.0.00.20 using default parameters. Resulting contigs were analyzed by Glimmer 3 [40] for gene calling. Transfer RNA genes were predicted using tRNAscan-SE 1.23 [41] and ribosomal RNA genes were found by similarity searches. Sequences were initially annotated by comparison with currently annotated bacterial sequences present in NCBI's NR protein database. Metabolic reconstruction and Gene Ontology classification assignments were performed using ASGARD [42], using the UniRef100 database [43]. Other annotation features were predicted using several programs, namely: transmembrane domain, by TMHMM 2.0c [44]; signal peptide, by SignalP 3.0b [45]; protein secretion probability, by SecretomeP 2.0 [46]; COG similarities and Pfam domain composition, by rpsblast [47]; protein characteristics (isoelectric point, molecular weight, and charge) by pepstat [48]. The resulting annotation and sequence assemblies were uploaded to GBrowse [49] installations for visualization and analysis. Overall DNA sequence identity comparison of the two strains was performed using MUMmer 3.20 [50]. Determination of putative ortholog relationship (and therefore the determination of gene uniqueness) between the genes of the two strains was performed by OrthoMCL 2.0b6 [51].

\section{Antibiotic resistance assay}

G. vaginalis strains 5-1 and AMD were diluted to an $\mathrm{OD}_{600}=0.1 \mathrm{in} \mathrm{BHI}$ supplemented with $1 \%$ yeast extract, $2 \%$ gelatin, and $0.1 \%$ starch (sBHI) containing $1 \%$ glucose (sBHIg). Antibiotics were serially diluted 2-fold in $200 \mu \mathrm{L}$ sBHIg in mictotiter wells and $3 \mu \mathrm{L}$ of the bacterial suspension was added to each well. The microtiter plates were incubated anaerobically for 24 hours and the lowest concentrations of antibiotics that prevented visible bacterial growth were recorded.

\section{Electron Microscopy}

Bacteria grown overnight in sBHI supplemented with $10 \%$ human serum (sBHIs) were collected by centrifugation, washed in sterile deionized water, spotted onto formvar-coated 200-mesh copper grids (Electron Microscopy Sciences, Hatfield, PA), stained with $2 \%$ phosphotungstic acid, and analyzed using a Jeol JEM-1230 transmission electron microscope equipped with a Gatan UltraScan 4000SP 4K x4K CCD camera.

\section{Cytotoxicity}

Strains AMD and 5-1 were cultured in sBHIs, collected by centrifugation, and resuspended in PBS to an $\mathrm{OD}_{600}=$ 0.15 . ME-180 monolayers were cultured in 96 well plates to $\sim 90 \%$ confluence, the media was replaced with $100 \mu \mathrm{L}$ $1 \times$ PBS, and $100 \mu \mathrm{L}$ of the bacterial suspensions was added. To asses the contact dependence of G. vaginalisassociated cytotoxicity, ME-180 monolayers were cultured in 6 well plates, the media was replaced with $2 \mathrm{ml}$ of $1 \times$ PBS, a sterile transwell with pores of either $0.45 \mu \mathrm{M}$ or $8 \mu \mathrm{M}$ was inserted into a well, and $2 \mathrm{ml}$ of the bacterial suspension was added to the top portion of the transwell. 
The monolayers were monitored every hour by light microscopy for cytopathogenic changes, such as cell rounding, loss of adhesion, and disruption of the monolayer. Photos were taken using an Olympus CK2 microscope at magnifications of $100 \times$ and $400 \times$.

\section{RNA Isolation and Quantitative RT-PCR}

Overnight cultures of the G. vaginalis strains were subcultured 1:20 in $5 \mathrm{ml}$ of sBHIs and grown for $\sim 6$ hours anaerobically at $37^{\circ} \mathrm{C}$ to mid-exponential phase. The cells were collected and resuspended in $500 \mu \mathrm{l}$ of buffer RLT (Qiagen, Germantown, MD). The suspension was transferred to Lysing Matrix B tubes (MP Biomedicals, Solon, $\mathrm{OH})$ and $500 \mu \mathrm{l}$ of a 5:1 mixture of acid phenol:cholorform (Ambion, Austin, TX) was added. The cells were lysed using a FastPrep FP120 Instrument (Thermo Scientific, Waltham, MA) with settings of power 6 and duration of 40 seconds. Samples were centrifuged 5 minutes $20,817 \mathrm{rpm}$ at $4^{\circ} \mathrm{C}$, the upper layer was transferred to a new microfuge tube containing $500 \mu \mathrm{L}$ EtOH $(500 \mu \mathrm{l})$, the samples were mixed by inversion, and the RNA purification was performed using the RNeasy kit (Qiagen) according to manufacturer's instructions, and the RNA was eluted in $90 \mu \mathrm{l}$ of RNase-free water. DNA was removed using the Turbo DNA-free kit (Ambion). RNA was quantified by measurement of absorbance at 260 and $280 \mathrm{~nm}$. Synthesis of cDNA from $1 \mu \mathrm{g}$ RNA was carried out using the Tetro $^{\circ} \mathrm{cDNA}$ synthesis kit (Bioline, Taunton, $\mathrm{MA}$ ) and $1 \mu \mathrm{M}$ Gv16sc-REV: 5'AGGTACACTCACCCGAAAGC3', MH8: 5'GTTAATGGTGCGCGATTTGC 3', and MH6: 5'GTTGTTAAAGAACACATCGAAG3'. Resulting cDNA was diluted 1:100, and $2 \mu \mathrm{l}$ of the diluted cDNA was used as a template for realtime RT-PCR in reactions that included $0.4 \mu \mathrm{M}$ of each forward and reverse primer as well as Sensimix Plus + Fluorescein (Quantace, Norwood, MA) at a final concentration of $1 \times$. Primer sets were as follows: $16 \mathrm{~S}$ - Gv16sc-FWD: 5'CACATTGGGACTGAGATACGG3' and Gv16sc-REV; vly - MH7: 5'CTTGCGCAGCCAGCAAGG3' and MH8; bapL - MH5: 5'GTGTCATTGAGCACACTTGC3' and MH6. Control reactions lacked reverse transcriptase enzyme to ensure that DNA contamination was minimal. Reactions were performed on an IQ5 Multicolor Realtime PCR Detection System (Bio-Rad, Hercules, CA). Optimal hybridization temperatures were determined by performing reactions over a $10^{\circ} \mathrm{C}$ gradient. Primer efficiencies were determined by using five, 5 -fold dilutions of cDNA as template and the resulting $\mathrm{Ct}$ values were plotted versus the $\log 10$ of the dilution factor, yielding a straight line, the slope of which was then used in the following equation: Efficiency $=10(1 / \mathrm{m})$ where $\mathrm{m}=$ slope. Reactions were incubated for 10 minutes at $95^{\circ} \mathrm{C}$ then cycled for 35 rounds of 10 seconds at $95^{\circ} \mathrm{C}, 10$ seconds at the determined hybridization temperature, and then $15 \mathrm{sec}-$ onds at $72^{\circ} \mathrm{C}$ during which data, SYBR green fluorescence, was collected. Data following RT-PCR was corrected for primer efficiency by applying the formula: Corrected $=$ EfficiencyCt. The corrected value obtained was normalized to $16 \mathrm{~s}$ rRNA expression. Averages were obtained from technical replicates and standard deviation was determined. Biological triplicates were performed for each sample.

\section{Adherence}

Bacteria were grown in sBHIs, and $\sim 10^{8}$ were collected by centrifugation and resuspended in $1 \times$ PBS. ME- 180 cervical epithelial cells (ATCC) were cultured at $37^{\circ} \mathrm{C}$ in $5 \%$ $\mathrm{CO}_{2}$ in McCoy's 5A medium (Quality Biologic, Gaithersburg, MD) supplemented with $10 \%$ fetal bovine serum and $1 \mathrm{IU} \mathrm{ml} \mathrm{ml}^{-1}$ penicillin/streptomycin (MediaTech, Manassass, VA) in 6-well polystyrene plates (Greiner, Monroe, NC) to $90 \%$ confluence. ME-180 cells were stained with $2.5 \mu \mathrm{M}$ Vybrant red membrane stain in PBS and bacteria were stained with a solution of $0.5 \mu \mathrm{M}$ BacLight green (Invitrogen, Carlsbad CA) in PBS at $37^{\circ} \mathrm{C}$ for 60 minutes. Following staining, epithelial cells and bacteria were washed twice in $1 \times$ PBS, the bacteria were added to the monolayer in a final volume of $3 \mathrm{~mL} P B S$, and the plates were centrifuged to maximize contact between the bacteria and epithelial cells. The bacteria were allowed to adhere at $4^{\circ} \mathrm{C}$ for 15 minutes, the monolayer was washed 3 times with $1 \times$ PBS, and adherence was visualized using a Zeiss LSM 510 META NLO multiphoton laser scanning microscope with an Achroplan $63 \times$ water dipping objective. BacLight green was excited with a $488 \mathrm{~nm}$ argon laser, Vybrant Red with a $633 \mathrm{nM}$ laser and emissions filters $515 \mathrm{~nm}$ (green) and $630 \mathrm{~nm}$ (red) were used.

\section{Biofilm formation}

Strains 5-1 and AMD were grown in sBHIs in 96 well tissue culture-treated plates (Greiner) anaerobically at $37^{\circ} \mathrm{C}$ for 24 hours. For a qualitative assessment of biofilm formation, non-adherent bacteria were removed from the wells and adherent bacteria were stained with safranin. For a quantitative measure of biofilm formation, planktonic bacteria were removed from the wells and transferred to new wells, and the biofilms were resuspended in fresh media. The $\mathrm{OD}_{595}$ was determined for both planktonic as well as biofilm growth. The percent growth as a biofilm was calculated as $\mathrm{OD}_{595}$ biofilm $/\left(\mathrm{OD}_{595}\right.$ biofilm + $\mathrm{OD}_{595}$ planktonic).

\section{Authors' contributions}

M.D.H. performed cytotoxicity, adhesion, biofilm, MIC, and qRT-PCR studies, performed electron microscopic analysis and wrote the paper. J.M.A. performed sequencing of $G$. vaginalis strains, subsequent anaylsis of results, and wrote the paper. G.A.B. contributed analytical tools required for completion of 
DNA sequencing and analyzed sequencing data. J.F.S. participated in the experimental design of the study and edited the manuscript. J.L.P. performed MIC, protein alignment, cytotoxicity, and qRT-PCR studies. A.T.O. performed cytotoxicity studies. P.H.G. contributed to research design and participated in editing of the manuscript. K.K.J. designed the research contained in this paper, analyzed and interpreted data, and edited the manuscript. All authors read and approved the final version of the manuscript.

\section{Competing interests}

The authors declare that they have no competing interests.

\section{Acknowledgements}

We are grateful to Drs. Andrew Onderdonk and Robin Ross, and to Mary Delaney (Harvard, Boston, MA) for providing G. vaginalis strains 5-1, 465, 101 and 551. Sequence analysis was performed in the Nucleic Acids Research Facilities at VCU, supported in part by funding from NIH_NCI 2P30 CA16059. Microscopy was performed at the VCU - Dept. of Neurobiology \& Anatomy Microscopy Facility, supported, in part, with funding from NIH-NINDS Center core grant (5P30NS047463). Supported in part by NIH grants 5P60MD002256, 5R01HD034612 and 1UH2AI083263.

\section{Author Details}

'Department of Microbiology and Immunology, Medical College of Virginia Campus of Virginia Commonwealth University, 1101 E. Marshall Street - PO Box 980678, Richmond, VA 23298 USA and 2Department of Obstetrics and Gynecology, Medical College of Virginia Campus of Virginia Commonwealth University, 1101 East Marshall Street - P.O. Box 980034, Richmond, VA 23298 USA

Received: 11 January 2010 Accepted: 11 June 2010

Published: 11 June 2010

\section{References}

1. Leopold S: Heretofore undescribed organism isolated from the genitourinary system. U S Armed Forces Med J 1953, 4(2):263-266

2. Gardner HL, Dukes CD: New etiologic agent in nonspecific bacterial vaginitis. Science 1954, 120(3125):853.

3. Gardner HL, Dukes CD: Haemophilus vaginalis vaginitis: a newly defined specific infection previously classified non-specific vaginitis. Am J Obstet Gynecol 1955, 69(5):962-976.

4. Greenwood JR, Pickett MJ: Salient features of Haemophilus vaginalis. J Clin Microbiol 1979, 9(2):200-204

5. Piot P, van Dyck E, Goodfellow M, Falkow S: A taxonomic study of Gardnerella vaginalis (Haemophilus vaginalis) Gardner and Dukes 1955. J Gen Microbiol 1980, 119(2):373-396.

6. Catlin BW: Gardnerella vaginalis: Characteristics, Clinical Considerations, and Controversies. Clin Microbiol Rev 1992, 5(3):213-237.

7. Sadhu K, Domingue PA, Chow AW, Nelligan J, Cheng N, Costerton JW: Gardnerella vaginalis has a gram-positive cell-wall ultrastructure and lacks classical cell-wall lipopolysaccharide. J Med Microbiol 1989, 29(3):229-235.

8. Criswell BS, Ladwig CL, Gardner HL, Dukes CD: Haemophilus vaginalis: vaginitis by inoculation from culture. Obstet Gynecol 1969, 33(2):195-199.

9. Ferris MJ, Masztal A, Aldridge KE, Fortenberry JD, Fidel PL Jr, Martin DH: Association of Atopobium vaginae, a recently described metronidazole resistant anaerobe, with bacterial vaginosis. BMC Infect Dis 2004, 4:5

10. Holst E, Svensson L, Skarin A, Westrom L, Mardh PA: Vaginal colonization with Gardnerella vaginalis and anaerobic curved rods. Scand J Urol Nephrol Suppl 1984, 86:147-152.

11. Swidsinski A, Mendling W, Loening-Baucke V, Ladhoff A, Swidsinski S, Hale LP, Lochs H: Adherent biofilms in bacterial vaginosis. Obstet Gynecol 2005, 106(5 Pt 1):1013-1023.

12. Gelber SE, Aguilar JL, Lewis KL, Ratner AJ: Functional and phylogenetic characterization of Vaginolysin, the human-specific cytolysin from Gardnerella vaginalis. J Bacteriol 2008, 190(11):3896-3903.

13. Reimer $L G$, Reller $L B$ : Gardnerella vaginalis bacteremia: a review of thirty cases. Obstet Gynecol 1984, 64(2):170-172.

14. Lagace-Wiens PR, Ng B, Reimer A, Burdz T, Wiebe D, Bernard K: Gardnerella vaginalis bacteremia in a previously healthy man: case report and characterization of the isolate. J Clin Microbio/ 2008, 46(2):804-806.

15. Amaya RA, Al-Dossary F, Demmler GJ: Gardnerella vaginalis bacteremia in a premature neonate. J Perinatol 2002, 22(7):585-587.

16. Graham S, Howes C, Dunsmuir R, Sandoe J: Vertebral osteomyelitis and discitis due to Gardnerella vaginalis. J Med Microbio/ 2009.

17. Hyman RW, Fukushima M, Diamond L, Kumm J, Giudice LC, Davis RW: Microbes on the human vaginal epithelium. Proc Natl Acad Sci USA 2005, 102(22):7952-7957.

18. Spiegel CA, Davick P, Totten PA, Chen KC, Eschenbach DA, Amsel R, Holmes KK: Gardnerella vaginalis and anaerobic bacteria in the etiology of bacterial (nonspecific) vaginosis. Scand J Infect Dis Supp/ 1983, 40:41-46.

19. Menard JP, Fenollar F, Henry M, Bretelle F, Raoult D: Molecular quantification of Gardnerella vaginalis and Atopobium vaginae loads to predict bacterial vaginosis. Clin Infect Dis 2008, 47(1):33-43.

20. Numanovic F, Hukic M, Nurkic M, Gegic M, Delibegovic Z, Imamovic A, Pasic S: Importance of isolation and biotypization of Gardnerella vaginalis in diagnosis of bacterial vaginosis. Bosn J Basic Med Sci 2008, 8(3):270-276.

21. Catlin BW: Gardnerella vaginalis: characteristics, clinical considerations, and controversies. Clin Microbiol Rev 1992, 5(3):213-237.

22. Reddy SP, Rasmussen WG, Baseman JB: Molecular cloning and characterization of an adherence-related operon of Mycoplasma genitalium. J Bacteriol 1995, 177:(20):5943-5951.

23. Boustouller YL, Johnson AP, Taylor-Robinson D: Pili on Gardnerella vaginalis studied by electronmicroscopy. J Med Microbio/ 1987, 23(4):327-329.

24. Johnson AP, Davies HA: Demonstration by electron microscopy of pili on Gardnerella vaginalis. Br J Vener Dis 1984, 60(6):396-397.

25. Wilson J: Managing recurrent bacterial vaginosis. Sex Transm Infect 2004, 80(1):8-11.

26. Kharsany AB, Hoosen AA, Van den Ende J: Antimicrobial susceptibilities of Gardnerella vaginalis. Antimicrob Agents Chemother 1993, 37(12):2733-2735.

27. Roberts MC, Hillier SL, Hale J, Holmes KK, Kenny GE: Tetracycline resistance and tetM in pathogenic urogenital bacteria. Antimicrob Agents Chemother 1986, 30(5):810-812.

28. National Committee for Clinical Laboratory Standards, Methods for antimicrobial susceptibility testing of anaerobic bacteria. Approved standard M11-A5, National Committee for Clinical Laboratory Standards, Wayne, PA. 2001.

29. Peixoto F, Camargos A, Duarte G, Linhares I, Bahamondes L, Petracco A: Efficacy and tolerance of metronidazole and miconazole nitrate in treatment of vaginitis. Int J Gynaecol Obstet 2008, 102(3):287-292.

30. Edwards DI: Nitroimidazole drugs--action and resistance mechanisms. II. Mechanisms of resistance. J Antimicrob Chemother 1993, 31(2):201-210.

31. Wastfelt M, Stalhammar-Carlemalm M, Delisse AM, Cabezon T, Lindahl G: Identification of a family of streptococcal surface proteins with extremely repetitive structure. J Bio/ Chem 1996, 271(31):18892-18897.

32. Madden JC, Ruiz N, Caparon M: Cytolysin-mediated translocation (CMT): a functional equivalent of type III secretion in gram-positive bacteria. Cell 2001, 104(1):143-152.

33. Latasa C, Solano C, Penades JR, Lasa I: Biofilm-associated proteins. $C R$ Biol 2006, 329(11):849-857.

34. Latasa C, Roux A, Toledo-Arana A, Ghigo JM, Gamazo C, Penades JR, Lasa I: BapA, a large secreted protein required for biofilm formation and host colonization of Salmonella enterica serovar Enteritidis. Mol Microbiol 2005, 58(5):1322-1339.

35. Hall-Stoodley L, Nistico L, Sambanthamoorthy K, Dice B, Nguyen D, Mershon WJ, Johnson C, Hu FZ, Stoodley P, Ehrlich GD, et al. Characterization of biofilm matrix, degradation by DNase treatment and evidence of capsule downregulation in Streptococcus pneumoniae clinical isolates. BMC Microbio/ 2008, 8:173.

36. Patterson $J$, Girerd PH, Karjane NW, Jefferson KK: Effect of biofilm phenotype on resistance of Gardnerella vaginalis to hydrogen peroxide and lactic acid. Am J Obstet Gynecol 2007, 197(2):170 e171-177.

37. Swidsinski A, Mendling W, Loening-Baucke V, Swidsinski S, Dorffel Y, Scholze J, Lochs H, Verstraelen H: An adherent Gardnerella vaginalis biofilm persists on the vaginal epithelium after standard therapy with oral metronidazole. Am J Obstet Gynecol 2008, 198(1):97 e91-96. 
38. Onderdonk AB, Lee ML, Lieberman E, Delaney ML, Tuomala RE: Quantitative microbiologic models for preterm delivery. J Clin Microbiol 2003, 41(3):1073-1079.

39. Patterson JL, Stull-Lane A, Girerd PH, Jefferson KK: Analysis of adherence, biofilm formation, and cytotoxicity suggest a greater virulence potential of Gardnerella vaginalis relative to other bacterial vaginosisassociated anaerobes. Microbiology 2009.

40. Salzberg SL, Pertea M, Delcher AL, Gardner MJ, Tettelin H: Interpolated Markov models for eukaryotic gene finding. Genomics 1999, 59(1):24-31

41. Lowe TM, Eddy SR: tRNAscan-SE: a program for improved detection of transfer RNA genes in genomic sequence. Nucleic Acids Res 1997, 25(5):955-964.

42. Alves JM, Buck GA: Automated system for gene annotation and metabolic pathway reconstruction using general sequence databases. Chem Biodivers 2007, 4(11):2593-2602.

43. Suzek BE, Huang H, McGarvey P, Mazumder R, Wu CH: UniRef: comprehensive and non-redundant UniProt reference clusters. Bioinformatics 2007, 23(10):1282-1288.

44. Krogh A, Larsson B, von Heijne G, Sonnhammer EL: Predicting transmembrane protein topology with a hidden Markov model: application to complete genomes. J Mol Bio/ 2001, 305(3):567-580.

45. Bendtsen JD, Nielsen H, von Heijne G, Brunak S: Improved prediction of signal peptides: SignalP 3.0. J Mol Biol 2004, 340(4):783-795.

46. Bendtsen JD, Kiemer L, Fausboll A, Brunak S: Non-classical protein secretion in bacteria. BMC Microbiol 2005, 5:58.

47. Altschul SF, Madden TL, Schaffer AA, Zhang J, Zhang Z, Miller W, Lipman DJ: Gapped BLAST and PSI-BLAST: a new generation of protein database search programs. Nucleic Acids Res 1997, 25(17):3389-3402.

48. Rice P, Longden I, Bleasby A: EMBOSS: the European Molecular Biology Open Software Suite. Trends Genet 2000, 16(6):276-277.

49. Stein LD, Mungall C, Shu S, Caudy M, Mangone M, Day A, Nickerson E, Stajich JE, Harris TW, Arva A, et al:: The generic genome browser: a building block for a model organism system database. Genome Res 2002, 12(10):1599-1610.

50. Kurtz S, Phillippy A, Delcher AL, Smoot M, Shumway M, Antonescu C, Salzberg SL: Versatile and open software for comparing large genomes. Genome Biol 2004, 5(2):R12.

51. Li L, Stoeckert CJ Jr, Roos DS: OrthoMCL: identification of ortholog groups for eukaryotic genomes. Genome Res 2003, 13(9):2178-2189.

doi: 10.1186/1471-2164-11-375

Cite this article as: Harwich et al., Drawing the line between commensal and pathogenic Gardnerella vaginalis through genome analysis and virulence studies BMC Genomics 2010, 11:375

\section{Submit your next manuscript to BioMed Central} and take full advantage of:

- Convenient online submission

- Thorough peer review

- No space constraints or color figure charges

- Immediate publication on acceptance

- Inclusion in PubMed, CAS, Scopus and Google Scholar

- Research which is freely available for redistribution

Submit your manuscript at www.biomedcentral.com/submit
C Biomed Central 